
340 .

5. - Markoff chains and Martin boundaries, Illinois J. Math. 4 (1960), 313-

6. R. S. Martin, Minimal positive harmonic functions, Trans. Amer. Math. Soc. 49 (1941), 137-172.

7. P. A. Meyer, Fonctionnelles multiplicatives et additive de Markov, Ann. Inst. Fourier (Grenoble) 12 (1962), 125-230.

8. D. Ray, Resolvents, transition functions and strongly Markovian processes, Ann. of Math. (2) 70 (1959), 43-78.

9. T. Watanabe, On the theory of Martin boundaries induced by countable Markov processes, Mem. Coll. Sci. Univ. Kyoto Ser. A 33 (1960), 39-108.

10. - On the equivalence of excessive functions and superharmonic functions in the theory of Markov processes. I, Proc. Japan Acad. 38 (1962), 397-401.

KYUSHU UNIVERSITY AND

UNIVERSITY OF ILLINOIS

\title{
A NONTRIVIAL NORMAL SUP NORM ALGEBRA
}

\section{BY ROBERT MCKISSICK ${ }^{1}$}

Communicated by I. M. Singer, December 17, 1962

Let $X$ be a compact Hausdorff space and $A$ a complex linear algebra of continuous complex-valued functions defined on $X$. Suppose $A$ is normal on $X$, i.e., for every pair of disjoint closed sets $K_{0}, K_{1}$ in $X$, there exists a function $f \in A$ such that $f\left(K_{0}\right)=0$ and $f\left(K_{1}\right)=1$. Does it follow that every continuous complex-valued function on $X$ can be uniformly approximated by functions in $A$ ? With the additional assumption that $A$ is closed under complex conjugation, it follows by the Stone-Weierstrass theorem. (Trivially, if $A$ is normal then $A$ separates points.) The same theorem implies that the analogous question in the case of real-valued functions has an affirmative answer. However, in the complex-valued case it need not be so. An example will be given which demonstrates this. In this example, the space $X$ is a suitably chosen compact set in the complex plane. The algebra is $R(X)$, the algebra of all functions which can be uniformly approximated on $X$ by rational functions whose poles lie outside $X$. It will be shown that $R(X)$ is normal on $X$ and is a proper sub-algebra of $C(X)$, the algebra of all continuous complex-valued functions on $X$. Since $R(X)$ is closed under uniform limits, this will be sufficient.

Two lemmas are needed to accomplish this. One is a modification of an observation of Mergelyan [1]. The second represents a slight extension of a result due to Beurling [2].

1 The research in this paper forms part of the author's doctoral dissertation, submitted to the Massachusetts Institute of Technology, Spring, 1963. 
LEMma 1. Let $\left\{\Delta_{i}\right\}$ be a sequence of open discs in the complex plane such that $\sum_{i}$ (radius $\left.\Delta_{i}\right)<1$. If

then $R(X) \neq C(X)$.

$$
X=\{z:|z| \leqq 1\}-\bigcup_{i=1}^{\infty} \Delta_{i}
$$

Proof. It suffices to demonstrate the existence of a finite complex Baire measure $\mu$ on $X$ which is orthogonal to every function in $R(X)$ and is not the zero measure.

Let $M$ be the space of finite complex Baire measures on the closed unit disc. Let

$$
X_{n}=\{z:|z| \leqq 1\}-\bigcup_{i=1}^{n} \Delta_{i}
$$

The boundary of $X_{n}, \partial X_{n}$, is the sum of a finite number of arcs of circles. Define $\mu_{n} \in M$ to be the measure which coincides with $d z$ on $\partial X_{n}$ and is zero elsewhere. Since the length of $\partial X_{n}$ is less than $4 \pi$, the total variation of $\mu_{n}$ certainly satisfies $\left\|\mu_{n}\right\| \leqq 4 \pi$. Thus the sequence $\left\{\mu_{n}\right\}$ has a subsequence which converges in the weak-star topology on $M$ to a measure $\mu$. It is easily verified that $\mu$ is supported on $X$, the intersection of the $X_{n}$.

Let $r$ be a rational function whose poles lie outside $X$. There exists a positive integer $N=N(r)$ such that the poles of $r$ lie outside $X_{N}$. By the Cauchy integral theorem

$$
\int_{|z| \geqq 1} r d \mu_{n}=\int_{\partial X_{n}} r d z=0, \quad \text { for } n \geqq N .
$$

Since $\mu$ is the weak-star limit of a subsequence of $\left\{\mu_{n}\right\}$

$$
\int_{|z| \leqq 1} r d \mu=\int_{X} r d \mu=0 .
$$

This implies that $\mu$ is orthogonal to $R(X)$.

Let $A_{n}$ be the area of $X_{n}$ and $A$ the area (Lebesgue measure) of $X$. By Green's theorem,

$$
\int_{|z| \leqq 1} \bar{z} d \mu_{n}=\int_{\partial X_{n}} \bar{z} d z=2 i A_{n} .
$$

Therefore,

$$
\int_{|z| \S 1} \bar{z} d \mu=\int_{X} \bar{z} d \mu=2 i \lim _{n \rightarrow \infty} A_{n}=2 i A .
$$


But

$$
A \geqq \pi\left[1-\sum_{i}\left(\operatorname{rad} \Delta_{i}\right)^{2}\right] \geqq \pi\left[1-\sum_{i}\left(\operatorname{rad} \Delta_{i}\right)\right]>0 .
$$

Thus $\mu$ is not the zero measure.

Lemma 2. Let $D$ be an open disc in the complex plane. For any $\epsilon>0$, there exists a sequence $\left\{\Delta_{k}\right\}$ of open discs contained in $D$ and a sequence $\left\{r_{n}\right\}$ of rational functions such that:

(i) $\sum_{k}\left(\operatorname{rad} \Delta_{k}\right)<\epsilon$.

(ii) The poles of $r_{n}$ lie in $\bigcup_{k=1}^{n} \Delta_{k}$.

(iii) The sequence $\left\{r_{n}\right\}$ converges uniformly on the complement of $\bigcup_{k=1}^{\infty} \Delta_{k}$ to a function which is identically zero outside $D$ and is nowhere zero on $D-\bigcup_{k=1}^{\infty} \Delta_{k}$.

Proof. It suffices to prove this when $D$ is the open unit disc. The proof, as mentioned earlier, rests heavily on a construction due to Beurling [2]. This construction will now be described.

The infinite product

$$
\phi(z)=\prod_{2}^{\infty}\left(1-\left(e^{1 / \log n z}\right)^{n}\right)
$$

converges uniformly in any $\operatorname{disc}|z| \leqq R<1$. Let $\alpha_{1}, \alpha_{2}, \cdots, \alpha_{k}, \cdots$ be the zeroes of $\phi(z)$ arranged so that $\left|\alpha_{k}\right| \leqq\left|\alpha_{k+1}\right|$. Let $m_{R}$ be the minimum value of $|\phi(z)|$ on the circle $|z|=R$. As Beurling proved, there is a sequence of circles $\left\{|z|=R_{n}\right\}$ such that $m_{R_{n}}$ tends to infinity as $R_{n}$ tends to one. Also, for $k$ sufficiently large, the derivative $\phi^{\prime}(z)$ satisfies

$$
\left|\frac{1}{\phi^{\prime}\left(\alpha_{k}\right)}\right|<e^{-\sqrt{ } k}
$$

Using these properties of $\phi(z)$, it is established by straightforward application of the Cauchy integral theorem that

$$
\sum_{k=1}^{\infty} \frac{1}{\phi^{\prime}\left(\alpha_{k}\right)\left(z-\alpha_{k}\right)}=\frac{1}{\phi(z)} \quad \text { for }|z|<1, z \neq \alpha_{k}
$$

and

$$
\sum_{k=1}^{\infty} \frac{1}{\phi^{\prime}\left(\alpha_{k}\right)\left(z-\alpha_{k}\right)} \equiv 0 \quad \text { for }|z| \geqq 1 \text {. }
$$

Given this result of Beurling, it remains to show that a sequence 
$\left\{\Delta_{k}\right\}$ of open discs centered at the $\alpha_{k}$ can be chosen so that the series

$$
\sum_{k=1}^{\infty} \frac{1}{\phi^{\prime}\left(\alpha_{k}\right)\left(z-\alpha_{k}\right)}
$$

converges uniformly on the complement of $\bigcup_{k=1}^{\infty} \Delta_{k}$. Moreover each $\Delta_{k}$ must be contained in $D$ and condition (i) must be satisfied.

A simple computation shows that, for $k$ sufficiently large, an open disc centered at $\alpha_{k}$ having radius $1 / k^{2}$ will be contained in $D$. Let $N$ be a positive integer such that this condition, as well as

$$
\left|\frac{1}{\phi^{\prime}\left(\alpha_{k}\right)}\right|<e^{-\sqrt{ } k} \text {, }
$$

is satisfied when $k \geqq N$. Require also that

$$
\sum_{N}^{\infty} \frac{1}{k^{2}}<\frac{\epsilon}{2} \text {. }
$$

Such an $N$ certainly exists.

For $k \geqq N$, choose $\Delta_{k}$ so that

$$
\left(\operatorname{rad} \Delta_{k}\right)=\frac{1}{k^{2}} .
$$

For $1 \leqq k<N$, choose $\Delta_{k}$ so that it is contained in $D$ and

$$
\left(\operatorname{rad} \Delta_{k}\right)<\frac{\epsilon}{2 N} .
$$

If $z$ is any point in the complement of $\bigcup_{k=1}^{\infty} \Delta_{k}$ then

$$
\left|\sum_{N}^{\infty} \frac{1}{\phi^{\prime}\left(\alpha_{k}\right)\left(z-\alpha_{k}\right)}\right| \leqq \sum_{N}^{\infty}\left|\frac{1}{\phi^{\prime}\left(\alpha_{k}\right)\left(z-\alpha_{k}\right)}\right| \leqq \sum_{N}^{\infty} k^{2} e^{-\sqrt{ } k}<\infty .
$$

This establishes uniform convergence on the complement of $\bigcup_{k=1}^{\infty} \Delta_{k}$. q.e.d. Lemma 2.

Let $\left\{D_{m}\right\}$ be the sequence of all open discs in the plane centered at points with rational co-ordinates and having rational radii. For each $m$, in accordance with Lemma 2 , there exists a sequence $\left\{\Delta_{k}^{m}\right\}$ of open sub-discs of $D_{m}$ such that

$$
\sum_{k}\left(\operatorname{rad} \Delta_{k}^{m}\right)<\frac{1}{2^{m}}
$$

(Apply Lemma 2 letting $D=D_{m}, \epsilon=1 / 2^{m}$.) 
Consider now the sequence of open discs in the plane consisting of the $\Delta_{k}^{m}$ as $m$ and $k$ range over all positive integers. Clearly

$$
\sum_{m, k}\left(\operatorname{rad} \Delta_{k}^{m}\right)<1 .
$$

Let

$$
X=\{z:|z| \leqq 1\}-\underset{m, k}{\bigcup} \Delta_{k}^{m} .
$$

By Lemma $1, R(X)$ is a proper sub-algebra of $C(X)$. It remains to show that $R(X)$ is normal on $X$.

Let $k$ be any closed set in $X$ and $p$ any point in $X-k$. There is an $m$ such that $p \in D_{m}$ and $D_{m} \cap k=\varnothing$. Since the $\Delta_{k}^{m}$ were chosen in accordance with Lemma 2 , there exists an $f \in R(X)$ such that $f(p) \neq 0$ and $f(k)=0$. If a commutative Banach algebra with identity has this separability property on its maximal ideal space, i.e., separates an arbitrary closed set and a point not in that set, it is normal on its maximal ideal space [3, Lemma $25 \mathrm{C}]$. But for any compact $X$ in the plane, $R(X)$, with the sup norm

$$
\|f\|=\sup _{x \in X}|f(x)|,
$$

is a commutative Banach algebra with identity and it is well known that the maximal ideal space of $R(X)$ is $X$.

Much of the credit for this example belongs to Professor Kenneth Hoffman of the Massachusetts Institute of Technology. It was his conjecture originally that an example of this kind could be found and it was he who supplied many important ideas along the way. I wish to thank him for this. My thanks also go to Professor John Wermer of Brown University who first realized the significance of Beurling's result, from a function algebra point of view, and directed my attention to it.

\section{REFERENCES}

1. S. N. Mergelyan, Uniform approximations to functions of a complex variable, Uspehi Mat. Nauk 7 (1952), no. 2(48), 31-122; Amer. Math. Soc. Transl. 101 (1954), 19.

2. A. Beurling, Sur les fonctions limites quasi analytiques des fractions rationelles, Attonde Skandinaviska Matematikerkongressen, 1934, p. 208.

3. L. H. Loomis, An introduction to abstract harmonic analysis, Van Nostrand, New York, 1953.

BROWN UNIVERSITY 